\title{
Atomic Level Structural Modulations at the Negatively Charged Domain Walls in $\mathrm{BiFeO}_{3}$ Films
}

\author{
W. Y. Wang ${ }^{1}$, Y. L. Tang ${ }^{1}$, Y. L. Zhu ${ }^{1}$, Y. B. Xu ${ }^{1}$, Y. Liu ${ }^{1}$, Y. J. Wang ${ }^{1}$, S. Jagadeesh ${ }^{1}$, and X. L. Ma ${ }^{1,2}$ \\ 1. Shenyang National Laboratory for Materials Science, Institute of Metal Research, Chinese Academy \\ of Sciences, Wenhua Road 72, 110016 Shenyang, China \\ 2. School of Materials Science and Engineering, Lanzhou University of Technology, Langongping Road \\ 287, 730050 Lanzhou, China
}

Rhombohedral multiferroic $\mathrm{BiFeO}_{3}$ (BFO) possesses various kinds of domain walls (DWs) which feature $109^{\circ}, 180^{\circ}$ and $71^{\circ}$ configurations, respectively. Depending on the orientations of DWs, the dipole configurations beside DWs can either be 'head-to-tail', 'head-to-head' or 'tail-to-tail' which then introduce zero, net positive or net negative bound charges at the DWs, respectively. Since the finding of novel DW conduction in BFO, several emergent phenomena at DWs are confirmed, such as electrically tunable conductance, enhanced photovoltage and magnetoresistant effect [1-3]. Particularly, charged DWs (CDWs) in BFO exhibit electrically controlled photo-detection property which implies nonvolatile optoelectronic memory applications [3]. In addition, these CDWs are also known to exhibit pinning effects on the movement of DWs and thus induce polarization fatigue in BFO memories. Although a charge-accumulation driven T phase was identified at a $71^{\circ} \mathrm{CDW}$ with 'head-to-head' polarizations [4], atomic-scale mapping of tail-to-tail CDWs in BFO films is still so incomprehensive that the physical insights of the CDW phenomena are known little.

In our recent study, BFO thin films are epitaxially grown on $\mathrm{SrTiO}_{3}(\mathrm{STO})$ (001) and $\mathrm{GdScO}_{3}(\mathrm{GSO})$

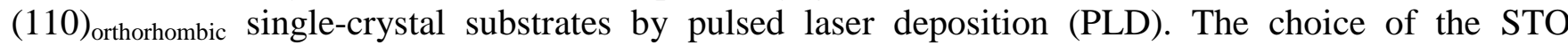
substrate is to introduce CDW by making use of the four-fold symmetry of the cubic structure in STO. The BFO films grown on GSO are further buffered with an epitaxial and conducting $\mathrm{SrRuO}_{3}(\mathrm{SRO})$ layer which promotes an introduction of $71^{\circ} \mathrm{CDW}$ through surface-compensating. Transmission electron microscopic samples are made by slicing, gluing, grinding and final ion milling with a Gatan PIPS 691. Aberration-corrected scanning transmission electron microscopic (AC-STEM) images are recorded using the high-angle angular dark-field (HAADF) detector on FEI Titan G2 60-300kV microscopy equipped with a high-brightness field-emission gun, double Cs correctors, and a monochromator operating at $300 \mathrm{kV}$. The probe convergence is $25 \mathrm{mrad}$ which yields a probe size of less than $1 \AA$. Before quantitative analysis, HAADF-STEM images are performed by fast Fourier transform (FFT) filtering using only a low-pass annular mask (slightly more than the resolution limit of the image). The atomic position is determined by two-dimensional Gaussian fitting of the individual atomic intensity profile of the STEM image using Matlab [5]. Two dimensional mapping of lattice spacing, lattice rotation and Fe sub-lattice displacement were also carried out using Matlab and Gatan DigitalMicrography software.

Using aberration-corrected scanning transmission electron microscopy (STEM), negatively CDWs are systematically investigated in BFO films, with their structures being directly mapped on the atomic scale. $109^{\circ}, 180^{\circ}$ and $71^{\circ}$ CDWs on (100) plane and a $109^{\circ} \mathrm{CDW}$ on (110) plane, with tail-to-tail dipole configurations, are thus revealed. Both the atomic structural details and sub-lattice ferroelectric ion displacements of these CDWs are quantitatively studied using aberration corrected HAADF-STEM with sub-angstrom precision. Lattice spacing $L x$ and lattice rotation Ry at the CDWs of (100) type show onedimensional modulations where alternate lattice expansions and clockwise/anticlockwise rotations are 
clearly imaged, which differ remarkably from both uncharged domain walls (UCDWs) and positively CDW with head-to-head polarizations in BFO reported previously. These findings are expected to broaden our understanding on DW structures as well as the effect of bound charges on structural stabilities of DWs [6,7].

\section{References:}

[1] Q He et al., Phys. Rev. Lett. 108 (2012), 067203.

[2] RK Vasudevan et al., Nano Lett. 12 (2012), P5524.

[3] WM Lee et al., Adv. Mater. 24 (2012), OP49.

[4] L Li et al., Nano Lett. 13 (2013), P5218.

[5] YL Tang et al., Science 348 (2015), P547.

[6] WY Wang et al., Adv. Mater. Interfaces (2015), DOI: 10.1002/admi.201500024.

[7] The authors acknowledge funding from the National Natural Science Foundation of China (No. 51231007, 51571197, 51501194, 51671194 and 51521091), National Basic Research Program of China (2014CB921002), and the Key Research Program of Frontier Sciences CAS (QYZDJ-SSW-JSC010). YL Tang acknowledges the IMR SYNL-T.S. Kê Research Fellowship and the Youth Innovation Promotion Association CAS (No. 2016177).
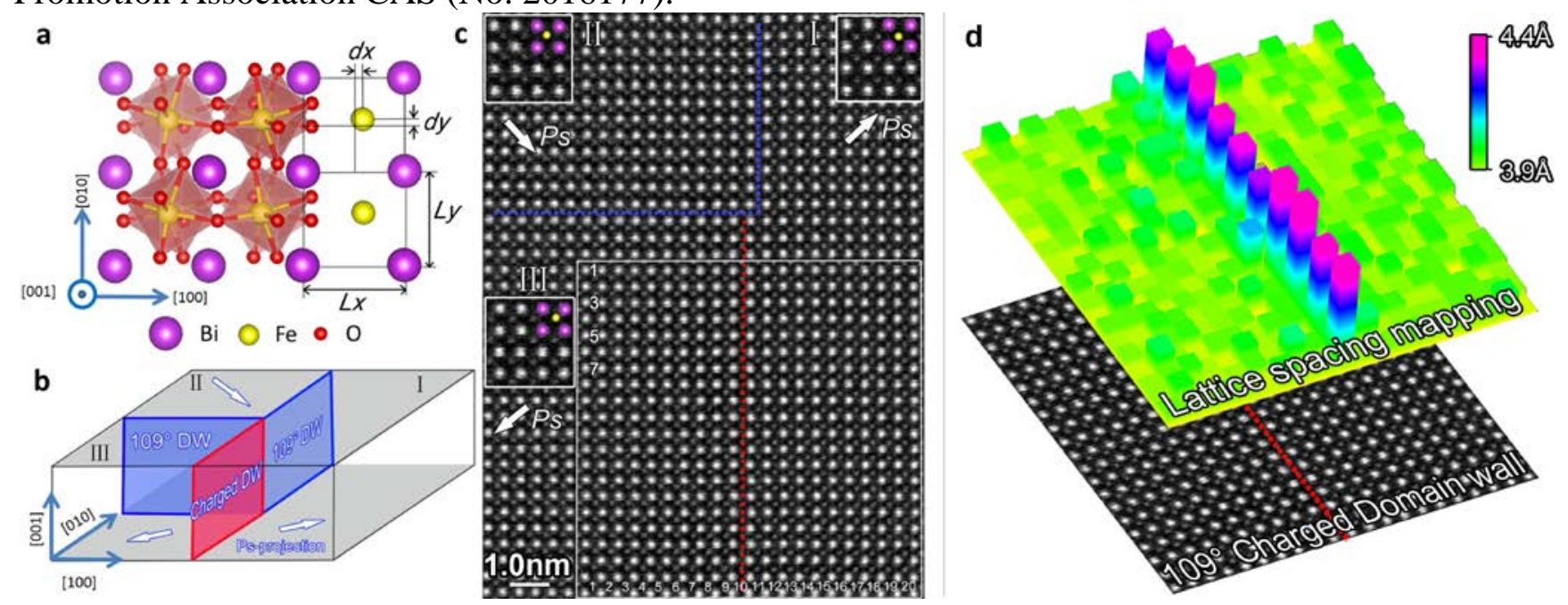

Figure 1. Illustration of $109^{\circ} \mathrm{CDW}$ of (100) type. (a) Atomic model of multiferroic BFO projected along [001] direction where $\mathrm{Bi}$, Fe and $\mathrm{O}$ are represented by violet, yellow, and red spheres, respectively. $d x$ and $d y$ are components of sub-lattice displacement vectors of Fe sub-lattice $D_{F}$ (from Bi sub-lattice center to Fe sub-lattice) in horizontal and vertical directions. $L x$ and $L y$ are the lattice spacing in horizontal and vertical directions. (b) Illustration of intersection of three domains I, II and III, the white arrows are the Ps projections of the corressponding domains along [001] direction. The blue planes are $109^{\circ}$ UCDWs of (100) type. The red plane is $109^{\circ} \mathrm{CDW}$ of (100) type. (c) A HAADF image of domain intersection projected along [001]. The $109^{\circ}$ UCDWs of (100) type is marked by blue dotted lines. A red dotted line indicates the $109^{\circ} \mathrm{CDW}$ of (100) type. The insets are the enlarged images of the corresponding area (violet: $\mathrm{Bi}$, yellow: Fe). The white arrows are the Ps projections. The square frame is the area used for analyzing the lattice variations and Fe sub-lattice displacements. (d) Atomic-level 1D modulation is demonstrated at the negatively charged domain walls in $\mathrm{BiFeO}_{3}$ thin films. All the $109^{\circ}$, $180^{\circ}$, and $71^{\circ}$ charged domain walls of (100) type reveal similar $1 \mathrm{D}$ modulations in terms of alternate lattice expansions and clockwise/counterclockwise lattice rotations [6]. 